\title{
Application of Speed-Gradient Variational Principle to Modeling Transient Processes in Thermodynamics *
}

\author{
Alexander L. Fradkov* \\ * Institute for Problems of Mechanical Engineering, Russian Academy of \\ Sciences, 61, Bolshoy ave. V.O., 199178, Saint Petersburg, Russia (Tel: +7 \\ 812 321-4766; e-mail: a.l.fradkov@ieee.org).
}

\begin{abstract}
The speed-gradient variational principle (SG-principle) is formulated and applied to thermodynamical systems. It is shown that Ziegler's Maximum Entropy Generation Principle as well as Prigogine's principle of minimum entropy production and Onsager's symmetry relations can be interpreted in terms of the SG-principle. For an SG thermodynamic system its negative entropy plays a role of the goal functional. The speed-gradient formulation of thermodynamic principles provides their extended versions, describing transient dynamics of nonstationary systems far from equilibrium. As an example an SG-model of transient (relaxation) dynamics for systems of a finite number of particles based on maximum entropy principle is derived. It has the form $d N(t) / d t=A \ln N(t)$, where $N(t)$ is the vector of the cell populations, $A$ is a symmetric matrix with two zero eigenvalues corresponding to mass and energy conservation laws.
\end{abstract}

Keywords: Nonequilibrium thermodynamics, variational principles, Onsager relations, Maximum Entropy Principle

\section{INTRODUCTION}

Variational principles are often used for building dynamical models both in physics and in engineering. In engineering variational principles are used to design equations of optimal control systems (Young, 1980). The equations of motion for physical systems are also frequently derived from variational principles: principle of least action, maximum entropy principle, etc. (Lanczos, 1964; Gyarmati, 1970). Although application of control methods in physics is a huge area of active recent research (Fradkov, 2007), there are only a few attempts to apply results of control theory to model physical systems (Rosenbrock, 2000).

In this paper the previous results (Fradkov, 2003) on using speed-gradient method of nonlinear and adaptive control for modeling physical systems are extended to nonequilibrium thermodynamics.

Variational principles are based on specification of a functional (usually, integral functional) and determination of real motions as points in an appropriate functional space providing extrema of the specified functional. The principle is called integral if the functional to be extremized has an integral form.

In addition to integral principles, differential (local) ones were proposed: Gauss principle of least constraint, principle of minimum energy dissipation and others. It has been pointed out by Plank (1914) that the local principles have some preference with respect to integral ones because they do not fix dependence of the current states and motions of the system on its later states and motions. In thermodynamics two of such principles have become most popular during last century: I. Prigogine's

^ The work was supported by the Research Program 22 of Presidium of RAS (project 1.8). The author thanks Prof. Tatiana Khantuleva for useful comments. principle of minimum entropy production and L. Onsager's symmetry principle for kinetic coefficients. Authors of both results were awarded with Nobel prizes. In the 1950s the so called Maximum Entropy Generation Principle (MEGP) was proposed by H. Ziegler (Ziegler, 1958, 1983) who also showed that Prigogine's and Onsager's principles in the near equilibrium case can be derived from MEGP under some conditions and that they are equivalent to each other. In 1957 E.T. Jaynes formulated the Maximum Entropy Principle (MEP): the entropy of any physical system tends to increase until it achieves its maximum value under constraints imposed by other physical laws (Jaynes, 1957). In fact such a prediction (implicit) can be found in the works of W. Gibbs.

In (Fradkov, 1990, 2003) a new local evolution principle, so called speed-gradient (SG) principle originated from the SGdesign method of nonlinear control theory (Fradkov, 1979; Fradkov and Pogromsky, 1998) was proposed and illustrated by a number of examples from mechanics. In (Fradkov, 2007) SGprinciple was extended to the case of systems with constraints.

This paper is devoted to application of the SG-principle to thermodynamics. First, the formulation of the SG-principle is recalled and some illustrating examples are presented. Then it is shown that Ziegler's MEGP as well as Prigogine's and Onsager's principles can be interpreted in terms of the SGprinciple. In all cases the entropy of the system plays a role of the goal functional. The speed-gradient formulation of thermodynamic principles provide their extended versions, suitable for the systems far from equilibrium. Moreover, it may describe their nonstationary, transient dynamics. In the paper the SGprinciple is applied to derivation of transient (relaxation) dynamics for a system driven by maximum entropy principle. 


\section{SPEED-GRADIENT VARIATIONAL PRINCIPLE}

Consider a class of physical systems described by systems of differential equations

$$
\dot{x}=f(x, u, t),
$$

where $x=\left(x_{1}, \ldots, x_{n}\right)^{T}$ is $n$-dimensional column vector of the system state ${ }^{T}$ is the transposition sign), $u=\left(u_{1}, \ldots, u_{m}\right)^{T}$ is $m$-dimensional column vector of free (input) variables, $\dot{x}=$ $d x / d t, t \geq 0$. The problem of modelling system dynamics can be posed as the search of a law of change of $u(t)$ meeting some criterion of "natural", or "reasonable" behavior of the system. Let such a behavior be specified as a tendency to achieve a goal, specified as decreasing the value of the goal functional $Q(x)$, where $Q(x)$ is given apriori. The choice of $Q(x)$ should reflect physical essence of the problem and it is critical for the result. An ultimate goal may be also introduced as achievement of the minimum value of $\mathrm{Q}(\mathrm{x})$ :

$$
Q(x(t)) \rightarrow 0 \text { as } t \rightarrow \infty,
$$

if a nonnegative $Q(x)$ is chosen: $Q(x) \geq 0$.

The first step of the speed-gradient procedure is to calculate the speed $\dot{Q}=\frac{d Q}{d t}=\omega(x, u, t)$, where $\omega(x, u, t)=\frac{\partial Q(x)}{\partial x} f(x, u, t)$. The second step is to evaluate the gradient of the speed $\nabla_{u} \dot{Q}$ with respect to input vector $u$ (speed-gradient vector). Finally the law of dynamics is formed as the feedback law in the finite form

or in the differential form

$$
u=-\gamma \nabla_{u} \dot{Q}(x, u, t) .
$$

$$
\frac{d u}{d t}=-\gamma \nabla_{u} \dot{Q}(x, u, t)
$$

where $\gamma>0$ is a positive scalar or a positive definite symmetric matrix gain (positivity of a matrix is understood as positive definiteness of associated quadratic form). The underlying idea of the choices (3) or (4) is that the motion along the anti-gradient of the speed $\dot{Q}$ provides decrease of $\dot{Q}$. It may eventually lead to negativity of $\dot{Q}$ which, in turn, yields decrease of $Q$. Under some natural assumptions achievement of the ultimate goal (2) can be derived as a mathematical statement (Fradkov, 1990; Fradkov and Pogromsky, 1998) which is, however, beyond the theme of this paper. Now the speed-gradient principle can be formulated as follows.

Speed-gradient principle: Among all possible motions of the system only those are realized for which the input variables change proportionally to the speed gradient $\nabla_{u} \dot{Q}(x, u)$ of an appropriate goal functional $Q(x)$. If there are constraints imposed on the system motion, then the speed-gradient vector should be projected onto the set of admissible (compatible with constraints) directions.

According to the SG-principle, to describe a system dynamics one needs to introduce the goal function $Q(x)$. The choice of $Q(x)$ should reflect the tendency of natural behavior to decrease the current value $Q(x(t))$. Systems obeying the SG-principle will be called $S G$-systems. In this paper only the models (1) in a special form are considered:

$$
\dot{x}=u,
$$

i.e. a law of change of the state velocities is sought.

Since gradient of a function is the direction of it maximum growth, the SG-direction is the direction of maximum growth for $\dot{Q}(x, u, t)$, i.e. direction of maximum production rate for $Q$. Respectively, the opposite direction corresponds to minimum production rate for $Q$. In the presence of constraints SGprinciple suggests that production rate for $Q$ is maximum under imposed constraints. The laws of dynamics under constraints can be found using the method of Lagrange multipliers.

The SG-laws with non-diagonal gain matrices $\gamma$ can be interpreted by introducing a non-Euclidean metric in the space of inputs is by means of the matrix $\gamma^{-1}$. The matrix $\gamma$ can be used to describe spatial anisotropy. Admitting dependence of the matrix $\gamma$ on $x$ one can recover dynamics law for complex mechanical systems described by Lagrangian or Hamiltonian formalism.

\section{EXAMPLES OF SPEED-GRADIENT LAWS OF DYNAMICS}

According to the speed-gradient principle, at first one needs to introduce the goal function $Q(x)$. The choice of $Q(x)$ should reflect the tendency of natural behavior to decrease the current value $Q(x(t))$. Let us consider a few illustrating examples.

Example 1. Motion of a particle in the potential field. In this case the vector $x=\left(x_{1}, x_{2}, x_{3}\right)^{T}$ consists of coordinates $x_{1}, x_{2}, x_{3}$ of a particle. Choose smooth $Q(x)$ as the potential energy of a particle and derive the speed-gradient law in the differential form. To this end, calculate the speed $\dot{Q}=$ $\partial Q / \partial x=\left[\nabla_{x} Q(x)\right]^{T} u$ and the speed-gradient $\nabla_{u} \dot{Q}=\nabla_{x} Q(x)$. Then, choosing the diagonal positive definite gain matrix $\Gamma=$ $m^{-1} I_{3}$, where $m>0$ is a parameter, $I_{3}$ is the $3 \times 3$ identity matrix, we arrive at the Newton's law $\dot{u}=-m^{-1} \nabla_{x} Q(x)$ or

$$
m \ddot{x}=-\nabla_{x} Q(x) .
$$

Note that the speed-gradient laws with nondiagonal gain matrices $\Gamma$ can be incorporated if a non-Euclidean metric in the space of inputs is introduced by the matrix $\Gamma^{-1}$. Admitting dependence of the metric matrix $\Gamma$ on $x$ one can obtain evolution laws for complex mechanical systems described by Lagrangian or Hamiltonian formalism.

The SG-principle applies not only to finite dimensional systems, but also to infinite dimensional (distributed) ones. Particularly, $x$ may be a vector of a functional space $\mathscr{X}$ and $f(x, u, t)$ may be a nonlinear differential operator (in such a case the solutions of (1) should be understood as generalized ones). We will omit mathematical details for simplicity.

Example 2. Wave, diffusion and heat transfer equations. Let $x=x(r), r=\operatorname{col}\left(r_{1}, r_{2}, r_{3}\right) \in \Omega$ be the temperature field or the concentration of a substance field defined in the domain $\Omega \subset \mathbb{R}^{3}$. Choose the goal functional evaluating nonuniformity of the field as follows

$$
Q_{t}(x)=\frac{1}{2} \int_{\Omega}\left|\nabla_{r} x(r, t)\right|^{2} d r,
$$

where $\nabla_{r} x(r, t)$ is the spatial gradient of the field and boundary conditions are assumed zero for simplicity. Calculation of the speed $\dot{Q}_{t}$ and then speed-gradient of $Q_{t}$, yields

$$
\dot{Q}_{t}=-\int_{\Omega} \Delta x(r, t) u(r, t) d r, \quad \nabla_{u} \dot{Q}_{t}=-\Delta x(r, t),
$$

where $\Delta=\sum_{i=1}^{3} \frac{\partial^{2}}{\partial r_{i}^{2}}$ is the Laplace operator. Therefore the speedgradient law in differential form (4) is 


$$
\frac{\partial^{2}}{\partial t^{2}} x(r, t)=-\gamma \Delta x(r, t)
$$

which corresponds to the D'Alembert wave equation. The SGlaw in finite form (3) reads

$$
\frac{\partial x}{\partial t}(t)=-\gamma \Delta x(r, t)
$$

and coincides with the diffusion or heat transfer equation.

Note that the differential form of the speed-gradient laws corresponds to reversible processes while the finite form generates irreversible ones. For modeling more complex dynamics a combination of finite and differential SG-laws may be useful.

In a similar way dynamical equations for many other mechanical, electrical and thermodynamic systems can be recovered. The SG-principle applies to a broad class of physical systems subjected to potential and/or dissipative forces.

\section{GENERALIZED ONSAGER RELATIONS}

The speed-gradient approach provides a new insight for various physical facts and phenomena. For example, we will give evidence for an extended version of the symmetry principle for kinetic coefficients (Onsager principle) in thermodynamics (Glansdorff and Prigogine, 1971) (it is also called the MaxwellBetti theorem in elasticity theory). Consider an isolated physical system whose state is characterized by a set of variables (thermodynamic parameters) $\xi_{1}, \xi_{2}, \ldots, \xi_{n}$. Let $x_{i}=\xi_{i}-\xi_{i}^{*}$ be deviations of the variables from their equilibrium values $\xi_{1}^{*}, \xi_{2}^{*}, \ldots, \xi_{n}^{*}$. Let the dynamics of the vector $x_{1}, x_{2}, \ldots, x_{n}$ be described by the differential equations

$$
\dot{x}_{i}=u_{i}\left(x_{1}, x_{2}, \ldots, x_{n}\right), \quad i=1,2, \ldots, n .
$$

Traditionally in the thermodynamical analysis the variables are split into two groups according to decomposition of the entropy production $\sigma=\sum_{i} X_{i} J_{i}$, where $X_{i}$ are thermodynamic forces, $J_{i}$ are thermodynamic flows (conjugate variables). Then the relations $\frac{\partial \sigma}{\partial J_{i}}=X_{i}, \frac{\partial \sigma}{\partial X_{i}}=J_{i}$ may significantly simplify further analysis. For our purposes such a decomposition is not needed and we will not use it in the paper.

Linearize equations (10) near equilibrium

$$
\dot{x}_{i}=-\sum_{k=1}^{n} \lambda_{i k} x_{k}, \quad i=1,2, \ldots, n .
$$

The Onsager's principle (Glansdorff and Prigogine, 1971) claims that the values $\lambda_{i k}$ (kinetic coefficients) satisfy the equations

$$
\lambda_{i k}=\lambda_{k i}, \quad i, k=1,2, \ldots, n .
$$

Recall that, initially Onsager was dealing only with the variables which are even with respect to the microscopic change of time ( $\alpha$-variables in the terminology of De Groot and Masur (1962). Later it was extended to more general case which however will not be considered here for the sake of simplicity.

In general, the Onsager principle is not valid for all systems e.g. for systems far from equilibrium. Its existing proofs (Landau and Lifshitz, 1980) require additional postulates. Below a simple new proof is given, showing that it is valid for irreversible speed-gradient systems without exceptions.

First of all, the classical formulation of the Onsager principle (12) should be extended to nonlinear systems. A natural extension is the following set of identities:

$$
\frac{\partial u_{i}}{\partial x_{k}}\left(x_{1}, x_{2}, \ldots, x_{n}\right)=\frac{\partial u_{k}}{\partial x_{i}}\left(x_{1}, x_{2}, \ldots, x_{n}\right)
$$

Obviously, for the case when the system equations (10) have linear form (11) the identities (13) coincide with (12). However, since linearization is not used in the formulation (13) there is a hope that the extended version of the Onsager law holds for some nonlinear systems far from equilibrium. The following statement specifies a class of systems for which this hope comes true.

There exists a smooth function $Q(x)$ such that equations (10) represent the speed-gradient law in finite form for the goal function $Q(x)$ if and only if the identities (13) hold for all $x_{1}, x_{2}, \ldots, x_{n}$.

The proof is very simple. Since (10) is the speed-gradient law for $Q(x)$, its right-hand sides can be represented in the form $u_{i}=-\gamma \frac{\partial \dot{Q}}{\partial u_{i}}, \quad i=1,2, \ldots, n$. Therefore $u_{i}=-\gamma\left(\partial Q / \partial x_{i}\right)$ (in view of $\left.\dot{Q}=\left(\nabla_{x} Q\right)^{T} u\right)$. Hence $\frac{\partial u_{i}}{\partial x_{k}}=-\gamma \frac{\partial^{2} Q}{\partial x_{i} \partial x_{k}}=\frac{\partial u_{k}}{\partial x_{i}}$, and identities (13) are valid. According to the standard result from the calculus, the condition (13) is necessary and sufficient for potentiality of the vector-field of the right-hand sides of (10), i.e. for existence of a scalar function $\bar{Q}$ such that $u_{i}=\gamma \nabla_{x} \bar{Q}=$ $\gamma \nabla_{u} \dot{\bar{Q}}$

Thus, for SG-systems the extended form of the Onsager equations (13) hold without linearization, i.e., they are valid not only near the equilibrium state. In a special case the condition (13) was proposed in (Farkas and Noszticzius, 1971). The theorem means that generalized Onsager relations (13) are necessary and sufficient for the thermodynamics system to obey the SGprinciple for some $\bar{Q}$. On the other hand, it is known that different potential functions for the same potential vector-field can differ only by a constant: $\bar{Q}=Q+$ const and their stationary sets coincide. In the formulation of the SG-principle in Sec. II the goal was introduced as minimization of $Q$. Since the entropy of the thermodynamical system increases according to the 2 nd Law, the natural goal function for thermodynamical systems is negative entropy $(-S)$. Therefore, if the system tends to maximize its entropy and the negative entropy serves as the goal function for the SG-evolution law, then at every time instant the direction of parameter change (e.g. change of thermodynamical flows for fixed thermodynamical forces) coincides with the direction maximizing the rate of entropy change. In turn, it coincides with the gradient of the entropy rate, i.e. we recover Ziegler's MEGP. It follows from MEGP (Ziegler, 1983; (Martyushev and Seleznev, 2006) that for system in a weakly nonequilibrium state (some of the thermodynamical forces become free), the system will be adjusting them in order to minimize entropy production (rate). That is, if Prigogine principle holds then the generalized Onsager principle (13) holds and vice versa. Note that for special case the relation between Prigogine principle and Onsager principle was established by Gyarmati (1970).

For the SG-systems some additional properties can be derived. Let for example a system be governed by SG-law with a convex entropy goal function $-S$. Then the decrease of the entropy production $\dot{S}$ readily follows from the identities $\ddot{S}=d \dot{S} / d t=$ $\left(\nabla_{x} \dot{S}\right)^{T} \dot{x}=\gamma\left(\nabla_{x}\left\|\nabla_{x} S\right\|^{2}\right)^{T} \nabla_{x} S=2 \gamma\left(\nabla_{x} S\right)^{T}\left[\nabla_{x}^{2} S\right]\left(\nabla_{x} S\right)$. 
Since the entropy $S(x)$ is concave, its Hessian matrix $\nabla_{x}^{2} S$ is negative semidefinite: $\nabla_{x}^{2} S \leq 0$. Hence $\ddot{S}(x) \leq 0$ and $\dot{S}$ cannot increase.

\section{SPEED-GRADIENT ENTROPY MAXIMIZATION}

Let us underly that the speed-gradient principle provides an answer to the question: how the system will evolve? It differs from the principles of maximum entropy, maximum Fisher information, etc. providing and answer to the questions: where? and how far? Particularly, it means that SG-principle generates equations for the transient (nonstationary) mode rather than the equations for the steady-state mode of the system. It allows one to study nonequilibrium and nonstationary situations, stability of the transient modes, maximum deviations from the limit mode, etc. Let us illustrate this feature by example of entropy maximization problem.

According to the 2nd thermodynamics law and to the Maximum Entropy Principle of Gibbs-Jaynes the entropy of any physical system tends to increase until it achieves its maximum value under constraints imposed by other physical laws. Such a statement provides knowledge about the final distribution of the system states, i.e. about asymptotic behavior of the system when $t \rightarrow \infty$. However it does not provide information about the way how the system moves to achieve its limit (steady) state.

In order to provide motion equations for the transient mode employ the SG-principle. Assume for simplicity that the system consists of $N$ identical particles distributed over $m$ cells. Let $N_{i}$ be the number of particles in the $i$ th cell and the mass conservation law holds:

$$
\sum_{i=1}^{m} N_{i}=N
$$

Assume that the particles can move from one cell to another and we are interested in the system behavior both in the steady-state and in the transient modes. The answer for the steady-state case is given by the Maximum Entropy Principle: if nothing else is known about the system, then its limit behavior will maximize its entropy Jaynes (1957). Let the entropy of the system be defined as logarithm of the number of possible states:

$$
S=\ln \frac{N !}{N_{1} ! \cdots N_{m} !} .
$$

If there are no other constraints except normalization condition (14) it achieves maximum when $N_{i}^{*}=N / m$. For large $N$ an approximate expression is of use. Namely, if the number of particles $N$ is large enough, one may use the Stirling approximation $N_{i} ! \approx\left(N_{i} / e\right)^{N_{i}}$. Then

$$
S \approx N \ln \frac{N}{e}-\sum_{i=1}^{m} N_{i} \ln \frac{N_{i}}{e}=-\sum_{i=1}^{m} N_{i} \ln \frac{N_{i}}{N}
$$

which coincides with the standard definition for the entropy $S=-\sum_{i=1}^{m} p_{i} \ln p_{i}$, modulo a constant multiplier $N$, if the probabilities $p_{i}$ are understood as frequencies $N_{i} / N$.

To get an answer for transient mode apply the SG-principle choosing the entropy $S(X)=-\sum_{i=1}^{m} N_{i} \ln N_{i}$ as the goal function to be maximized, where $X=\operatorname{col}\left(N_{1}, \ldots, N_{m}\right)$ is the state vector of the system. Assume for simplicity that the motion is continuous in time and the numbers $N_{i}$ are changing continuously, i.e. $N_{i}$ are not necessarily integer (for large $N_{i}$ it is not a strong restriction). Then the sought law of motion can be represented in the form

$$
\dot{N}_{i}=u_{i}, i=1, \ldots, m,
$$

where $u_{i}=u_{i}(t), i=1, \ldots, m$ are controls - auxiliary functions to be determined. According to the SG-principle one needs to evaluate first the speed of change of the entropy (15) with respect to the system (16), then evaluate the gradient of the speed with respect to the vector of controls $u_{i}$ considered as frozen parameters and finally define actual controls proportionally to the projection of the speed-gradient to the surface of constraints (14). In our case the goal function is the entropy $S$ and its speed coincides with the entropy production $\dot{S}$. In order to evaluate $\dot{S}$ let us again approximate $S$ from the Stirling formula $N_{i} ! \approx\left(N_{i} / e\right)^{N_{i}}$ :

$$
\hat{S}=N \ln N-N-\sum_{i=1}^{m}\left(N_{i} \ln N_{i}-N_{i}\right)=N \ln N-\sum_{i=1}^{m} N_{i} \ln N_{i} .
$$

Evaluation of $\dot{\hat{S}}$ yields

$$
\dot{\hat{S}}=-\sum_{i=1}^{m}\left(\left(u_{i} \ln N_{i}+N_{i} \frac{u_{i}}{N_{i}}\right)=-\sum_{i=1}^{m} u_{i}\left(\ln N_{i}+1\right) .\right.
$$

It follows from (14) that $\sum_{i=1}^{m} u_{i}=0$. Hence $\dot{\hat{S}}=-\sum_{i=1}^{m} u_{i} \ln N_{i}$. Evaluation of the speed-gradient yields $\frac{\partial \dot{\hat{S}}}{\partial u_{i}}=-\ln N_{i}$ and the SG-law $u_{i}=\gamma\left(-\ln N_{i}+\lambda\right), \quad i=1, \ldots, m$, where Lagrange multiplier $\lambda$ is chosen in order to fulfill the constraint $\sum_{i=1}^{m} u_{i}=$ 0 , i.e. $\lambda=\frac{1}{m} \sum_{i=1}^{m} \ln N_{i}$. The final form of the system dynamics law is as follows:

$$
\dot{N}_{i}=\frac{\gamma}{m} \sum_{i=1}^{m} \ln N_{i}-\gamma \ln N_{i}, i=1, \ldots, m
$$

According to the SG-principle the equation (18) determines transient dynamics of the system. To confirm consistency of the choice (18) let us find the steady-state mode, i.e. evaluate asymptotic behavior of the variables $N_{i}$. To this end note that in the steady-state $\dot{N}_{i}=0$ and $\sum_{i=1}^{m} \ln N_{i}=\ln N_{i}$. Hence all $N_{i}$ are equal: $N_{i}=N / m$ which corresponds to the maximum entropy state and agrees with thermodynamics.

The next step is to examine stability of the steady-state mode. It can be done by means of the entropy Lyapunov function $V(X)=S_{\text {max }}-S(X) \geq 0$, where $S_{\max }=N \ln m$. Evaluation of $\dot{V}$ yields

$$
\dot{V}=-\dot{S}=\sum_{i=1}^{m} u_{i} \ln N_{i}=\frac{\gamma}{m}\left[\left(\sum_{i=1}^{m} \ln N_{i}\right)^{2}-m \sum_{i=1}^{m}\left(\ln N_{i}\right)^{2}\right] .
$$

It follows from the Cauchy-Bunyakovsky-Schwarz inequality that $\dot{V}(X) \leq 0$ and the equality $\dot{V}(X)=0$ holds if and only if all the values $N_{i}$ are equal, i.e. only at the maximum entropy state. Thus the law (18) provides global asymptotic stability of the maximum entropy state. The physical meaning of the law (18) is moving along the direction of the maximum entropy production rate (direction of the fastest entropy growth).

The case of more than one constraint can be treated in the same fashion. Let in addition to the mass conservation law (14) the energy conservation law hold. Let $E_{i}$ be the energy of the particle in the $i$ th cell and the total energy $E=\sum_{i=1}^{m} N_{i} E_{i}$ be conserved. The energy conservation law

$$
E=\sum_{i=1}^{m} N_{i} E_{i}
$$

appears as an additional constraint. Acting in a similar way, we arrive at the law (18) which needs modification to ensure conservation of the energy (19). According to the SG-principle 
one should form the projection onto the surface (in our case subspace of dimension $m-2$ ) defined by the relations

$$
\sum_{i=1}^{m} u_{i} E_{i}=0, \quad \sum_{i=1}^{m} u_{i}=0 .
$$

It means that the evolution law should have the form

$$
u_{i}=\gamma\left(-\ln N_{i}\right)+\lambda_{1} E_{i}+\lambda_{2}, i=1, \ldots, m,
$$

where $\lambda_{1}, \lambda_{2}$ are determined by substitution of (21) into (20). The obtained equations are linear in $\lambda_{1}, \lambda_{2}$ and their solution is given by formulas

$$
\left\{\begin{array}{l}
\lambda_{1}=\frac{\gamma m\left(\sum_{i=1}^{m} E_{i} \ln N_{i}\right)-\gamma\left(\sum_{i=1}^{m} E_{i}\right)\left(\sum_{i=1}^{m} \ln N_{i}\right)}{m \sum_{i=1}^{m} E_{i}^{2}-\left(\sum_{i=1}^{m} E_{i}\right)^{2}}, \\
\lambda_{2}=\frac{\gamma}{m} \sum_{i=1}^{m} \ln N_{i}-\frac{\lambda_{1}}{m} \sum_{i=1}^{m} E_{i} .
\end{array}\right.
$$

The solution of (22) is well defined if $m \sum_{i=1}^{m} E_{i}^{2}-\left(\sum_{i=1}^{m} E_{i}\right)^{2} \neq$ 0 which holds unless all the $E_{i}$ are equal (degenerate case).

Let us evaluate the equilibrium point of the system (16), (21) and analyze its stability. At the equilibrium point of the system the following equalities hold: $\gamma\left(-\ln N_{i}\right)+\lambda_{1} E_{i}+\lambda_{2}=0, i=$ $1, \ldots, m$. Hence

$$
N_{i}=C \exp \left(-\mu E_{i}\right), i=1, \ldots, m,
$$

where $\mu=\lambda_{1} / \gamma$ and $C=\exp \left(-\lambda_{2} / \gamma\right)$.

The value of $C$ can also be chosen from the normalization condition $C=N\left(\sum_{i=1}^{m} \exp \left(-\mu E_{i}\right)\right)$. We see that equilibrium of the system with conserved energy corresponds to the Gibbs distribution which agrees with classical thermodynamics. Again it is worth to note that the direction of change of the numbers $N_{i}$ coincides with the direction of the fastest growth of the local entropy production subject to constraints. As before, it can be shown that $V(X)=S_{\max }-S(X)$ is Lyapunov function for the system and that the Gibbs distribution is the only stable equilibrium of the system in nongenerate cases. Substitution of $\lambda_{1}, \lambda_{2}$ from (22) into equation (21) yields general form of evolution law for the frequencies in the form

$$
\frac{d}{d t} \bar{N}(t)=A \ln \bar{N}(t),
$$

where symmetric $m \times m$ matrix $A$ is defined as follows:

$$
a_{i j}=-\delta_{i j}+\frac{1}{m}+\tilde{E}_{i} \tilde{E}_{j}, i, j=1, \ldots, m
$$

$\delta_{i j}=1$, if $i=j, \delta_{i j}=0$, if $i \neq j, \tilde{E}_{i}=E_{i}-\frac{1}{m} \sum_{i=1}^{m} E_{i}$. It depends on the vector of energies $\left.\bar{E}=\left(E_{1}, \ldots, E_{m}\right)^{T}\right)$. According to its structure the matrix $A$ is symmetric and has two zero eigenvalues. Eq. (24) has a unique relative equilibrium corresponding to Gibbs distribution. Similar results are valid for continuous (distributed) systems even for more general problem of minimization of relative entropy (Kullback divergence) (Fradkov, 2007).

\section{CONCLUSIONS}

Speed-gradient variational principle provides a useful yet simple addition to classical results in thermodynamics. Whereas the classical results allow researcher to answer the question "Where it goes to?", the speed-gradient approach provides an answer to Fradkov and Pogromsky (1998) the question: "How it goes and how it reaches its steady-state mode?" SG-principle suggests that the transient behavior is potential with respect to the rate of some goal function. This idea may be applied to evaluation of nonequilibrium stationary states and study of system internal structure evolution (Khantuleva, 2005), description of transient dynamics of complex networks (Rangan and Cai, 2006; Boccaletti et al., 2006; Barabasi, 2007), description of transient slow motions in vibrational mechanics, etc. Other physical applications of techniques and ideas developed in control theory (cybernetics) can be found in (Sieniutycz, 1994; Gorban and Karlin, 2004; Fradkov, 2005, 2007).

\section{REFERENCES}

A. L. Barabasi. The architecture of complexity. IEEE Control Syst. Mag., pages 33-42, August 2007. (Special Section on Complex Networked Control Systems.

S. Boccaletti, V. Latora, Y. Morenod, M. Chavez, and D.U. Hwanga. Complex networks: Structure and dynamics. Physics Reports, 424:175-308, 2006.

S. R De Groot and P. Masur. Non-equilibrium Thermodynamics. North-Holland, Amsterdam, 1962.

H. Farkas and Z. Noszticzius. On the non-linear generalization of the gyarmati principle and theorem. Annalen der Physik, 27:341-348, 1971.

A. L. Fradkov. Speed-gradient scheme and its applications in adaptive control. Autom. Remote Control, 40(9):1333-1342, 1979.

A. L. Fradkov. Adaptive Control in Complex Systems. Nauka, Moscow, 1990. (in Russian).

A. L. Fradkov. Speed-gradient approach to modeling dynamics of physical systems. In Proc. Europ. Contr. Conf. (ECC'03), Cambridge, UK, September 1-4, 2003.

A. L. Fradkov. Application of cybernetical methods in physics. Physics-Uspekhi, 48(2):103-127, 2005.

A. L. Fradkov. Cybernetical physics: from control of chaos to quantum control. Springer-Verlag, Berlin - Heidelberg, 2007. 242 p.

A. L. Fradkov and A. Yu. Pogromsky. Introduction to control of oscillations and chaos. World Scientific, Singapore, 1998.

P. Glansdorff and I. Prigogine. Thermodynamics of Structure, Stability and Fluctuations. Wiley, New York, 1971.

A. N. Gorban and I. V. Karlin. Invariant manifolds for chemical and physical systems. In LN Physics, volume 600. SpringerVerlag, Berlin, Heidelberg, 2004.

I. Gyarmati. Non-equilibrium Thermodynamics. Field Theory and Variational Principles. Springer-Verlag, Berlin, 1970.

E. T. Jaynes. Information theory and statistical mechanics, I, II. Phys. Rev., 106, 108, 1957.

T. A. Khantuleva. Internal control in nonequilibrium transport. In Proc. 2nd IEEE-EPS Intern. Conf. Physics and Control, pages 41-46, St. Petersburg, 2005.

C. Lanczos. The Variational Principles of Mechanics. University Press, Toronto, 1964.

L. D. Landau and E. M. Lifshitz. Statistical Physics, Part 1. Pergamon Press, Oxford, 1980.

L. M. Martyushev and V. D. Seleznev. Maximum entropy production principle in physics, chemistry and biology. Physics Reports, 426(1):1-45, 2006.

M. Plank. Das prinzip der kleinsten wirkung. Die Kultur der Gegenwart, 3:692-702, 1914. Abt. 3, Bd. 1 (Also in: Physicalishe Abhandlungen und Vortrage, Bd.3, Braunshweig, 1958, ss. 91-101).

A. V. Rangan and D. Cai. Maximum-entropy closures for kinetic theories of neuronal network dynamics. Phys. Rev. Lett., 96:178101, 2006.

H. H. Rosenbrock. Doing quantum mechanics with control theory. IEEE Trans. Automat. Contr., 45(1):73-77, 2000. 
S. Sieniutycz. Conservation laws in variational thermohydrodynamics. Kluwer, Dordrecht, 1994.

L. C. Young. Lectures on the Calculus of Variations and Optimal Control Theory. Chelsea, New York, 1980. 2nd ed.

H. Z. Ziegler. Ang. Math. Phys., 9b:748, 1958.

H. Z. Ziegler. Introduction to Thermomechanics. NorthHolland, Amsterdam, 1983. 2nd ed. 